\title{
Genomic copy number variation association study in Caucasian patients with nonsyndromic cryptorchidism
}

Yanping Wang ${ }^{1}$, Jin Li $i^{3}$, Thomas F. Kolon ${ }^{4}$, Alicia Olivant Fisher ${ }^{1}$, T. Ernesto Figueroa ${ }^{2}$, Ahmad H. BaniHani ${ }^{2}$, Jennifer A. Hagerty², Ricardo Gonzalez ${ }^{2,9}$, Paul H. Noh ${ }^{2,10}$, Rosetta M. Chiavacci ${ }^{3}$, Kisha R. Harden ${ }^{3}$, Debra J. Abrams ${ }^{3}$, Deborah Stabley ${ }^{1}$, Cecilia E. Kim³ ${ }^{3}$, Katia Sol-Church ${ }^{1}$, Hakon Hakonarson ${ }^{3,5,6}$, Marcella Devoto ${ }^{5,6,7,8}$ and Julia Spencer Barthold ${ }^{1,2^{*}}$

\begin{abstract}
Background: Copy number variation (CNV) is a potential contributing factor to many genetic diseases. Here we investigated the potential association of CNV with nonsyndromic cryptorchidism, the most common male congenital genitourinary defect, in a Caucasian population.

Methods: Genome wide genotyping were performed in 559 cases and 1772 controls (Group 1) using Illumina HumanHap550 v1, HumanHap550 v3 or Human610-Quad platforms and in 353 cases and 1149 controls (Group 2) using the Illumina Human OmniExpress 12v1 or Human OmniExpress 12v1-1. Signal intensity data including log $R$ ratio (LRR) and B allele frequency (BAF) for each single nucleotide polymorphism (SNP) were used for CNV detection using PennCNV software. After sample quality control, gene- and CNV-based association tests were performed using cleaned data from Group 1 (493 cases and 1586 controls) and Group 2 (307 cases and 1102 controls) using ParseCNV software. Meta-analysis was performed using gene-based test results as input to identify significant genes, and CNVs in or around significant genes were identified in CNV-based association test results. Called CNVs passing quality control and signal intensity visualization examination were considered for validation using TaqMan CNV assays and QuantStudio ${ }^{\oplus}$ 3D Digital PCR System.

Results: The meta-analysis identified 373 genome wide significant $\left(p<5 \times 10^{-4}\right)$ genes/loci including 49 genes/loci with deletions and 324 with duplications. Among them, 17 genes with deletion and 1 gene with duplication were identified in CNV-based association results in both Group 1 and Group 2. Only 2 genes (NUCB2 and UPF2) containing deletions passed CNV quality control in both groups and signal intensity visualization examination, but laboratory validation failed to verify these deletions.
\end{abstract}

Conclusions: Our data do not support that structural variation is a major cause of nonsyndromic cryptorchidism.

Keywords: Cryptorchidism, Genetics, CNV

\footnotetext{
* Correspondence: Julia.Barthold@nemours.org

${ }^{1}$ Nemours Biomedical Research, Nemours /Alfred I. duPont Hospital for

Children, Wilmington, DE 19803, USA

${ }^{2}$ Division of Urology, Nemours/Alfred I. duPont Hospital for Children,

Wilmington, DE 19803, USA

Full list of author information is available at the end of the article
}

(c) The Author(s). 2016 Open Access This article is distributed under the terms of the Creative Commons Attribution 4.0 International License (http://creativecommons.org/licenses/by/4.0/), which permits unrestricted use, distribution, and reproduction in any medium, provided you give appropriate credit to the original author(s) and the source, provide a link to the Creative Commons license, and indicate if changes were made. The Creative Commons Public Domain Dedication waiver (http://creativecommons.org/publicdomain/zero/1.0/) applies to the data made available in this article, unless otherwise stated. 


\section{Background}

Nonsyndromic cryptorchidism, or isolated undescended testis, is one of the most common pediatric congenital anomalies, affecting $2-3 \%$ of boys, and is associated with infertility and testicular malignancy later in life [1]. The etiology is largely unknown and likely multifactorial. Familial clustering suggests moderate genetic contribution to the disease [2].

A candidate approach to gene discovery has revealed some potential risk genes, but the results are inconsistent and population-specific [3-10]. Recently we performed a genome-wide association study (GWAS) in 912 nonsyndromic cryptorchidism cases and 2921 controls $[11,12]$ to identify common allelic variants across the genome associated with the disease. No variant reached genome-wide significance $\left(p \leq 7 \times 10^{-9}\right)$ in full analysis, and one variant (rs55867206, near SH3PXD2B, $p=2 \times 10^{-9}$ ) passed this threshold in a subgroup analysis of proximal testis position. Pathway analysis of suggestive association markers $\left(p \leq 10^{-3}\right)$ using several bioinformatics tools identified overrepresentation of genes/functions linked to cytoskeleton-dependent processes, syndromic cryptorchidism and hypogonadotropic hypogonadism.

Over the past decade, evidence has shown that copy number variation $(\mathrm{CNV})$ plays an important role in the occurrence of many diseases [13]. Analysis of CNVs using array comparative genomic hybridization found VAMP7 duplication and OTX1 deletion in individuals with congenital genitourinary defects $[14,15]$, with cryptorchidism as one of the primary traits. However, the association of CNVs with nonsyndromic cryptorchidism has not been explored. Through analysis of GWAS data [11, 12], we hypothesized that $\mathrm{CNV}$ is a significant cause of nonsyndromic cryptorchidism in Caucasian males.

\section{Methods}

\section{Subjects and genotyping}

Cases were self-reported Caucasian subjects with nonsyndromic cryptorchidism who underwent surgical repair at Nemours/Alfred I. DuPont Hospital for Children (Nemours) or The Children's Hospital of Philadelphia (CHOP). Subjects with multiple congenital anomalies or diagnosis of any syndrome, other genital anomalies (hypospadias, chordee or other penile anomalies) or abdominal wall defects were excluded from the study. Control subjects were recruited through the CHOP Health Care Network. They were self-reported Caucasian males who were at least 6 years old with no known history of testicular disease, penile anomaly, diagnosis of a syndrome or any additional medical disorder associated with cryptorchidism. Basic demographic and phenotypic data collected include age of diagnosis, race, ethnicity, laterality and the position of affected testes.
As described in detail in previous publications [11, 12], two groups of cases were genotyped at the Center for Applied Genomics at CHOP to match available control genotype data. In Group 1, 559 cases and 1772 controls were genotyped using the Illumina HumanHap550 v1, HumanHap550 v3 or Human610-Quad platforms that share over $535 \mathrm{~K}$ single nucleotide polymorphisms (SNPs) in common. In Group 2, 353 cases and 1149 controls were genotyped using the Illumina Human OmniExpress 12v1 or Human OmniExpress 12v1-1 platforms that share over 719 K SNPs. The global SNP and gene coverage of our SNP arrays are approximately $85 \%$ and $80 \%$, respectively [16], and the average distance between probes is $4 \mathrm{kbp}-5.5$ kbp. At SNP genotype calling, cluster files (.egt) provided by Illumina were used as a common reference.

\section{CNV detection and sample quality control}

Due to differences in SNP coverage and less than $310 \mathrm{~K}$ intersection of SNPs between platforms used in the 2 case-control groups, CNV detection, sample quality control (QC), and association tests were performed separately in Groups 1 and 2. We used the PennCNV software package [17-20] to make CNV calls based on signal intensity data from genotyping arrays including $\log \mathrm{R}$ ratio (LRR) and B allele frequency (BAF) for each SNP. Adjacent CNV calls were then automatically examined and merged using PennCNV software.

We used sample QC criteria from our prior genomewide genotyping data analysis in PLINK [11, 12, 21-23]. Individuals were excluded from further analysis if one of below criteria were met: (1) discordance between reported sex and sex chromosome SNP data; (2) missing genotype rate $>3 \%$; (3) potential duplicates or relatives (based on estimate of proportion of alleles shared identical by descent $>0.1875$ ); and (4) non-Caucasian ancestry based on multidimensional scaling (MDS) analysis using data from the Stanford Human Genome Diversity Project (HGDP) [24, 25]. We removed all samples that deviated from the means of the first or second MDS components by more than 3 standard deviations (SD). We also used a sample quality control function implemented in the ParseCNV software package $[26,27]$ and removed samples with (1) high intensity noise (measured by SDLRR (SD of LRR) > mean $+3 \mathrm{SD}$ ); (2) extreme intensity waviness (measured by more than $3 \mathrm{SD}$ of mean of GCWF (Guanine-Cytosine base pair wave factor)) and (3) high number of CNV counts per sample (measured by $\mathrm{CNV}$ count number $>$ mean $+3 \mathrm{SD}$ ).

\section{Gene based association analysis, meta-analysis and CNV based association analyses}

Given that SNP overlap is low between the genotyping platforms used in Group 1 and 2, and the uncertainty of CNV boundary data from different platforms, we were unable to 
directly merge CNV from the two groups. Therefore, after removing individuals not passing samples QC, we performed gene-based association tests separately in Group 1 and 2 samples using the ParseCNV software package. We then performed meta-analyses of gene-based association results with METAL software $[28,29]$ using gene names as markers to identify significant genes $\left(p<5 \times 10^{-4}\right.$, a conservative bar for $\mathrm{CNV}$ genome-wide significance suggested by ParseCNV). We also performed CNV-based association tests in cleaned Group 1 and 2 samples using ParseCNV software package. CNVs in or around significant genes from the gene-based meta-analyses were identified by searching the "gene" column in CNV-based association tests results. The CNVs were considered not passing CNV $\mathrm{QC}$ and removed if one of below criteria were met: average number of probes in CNV (AvgProbes) $<5$, worst p-value in the span of $\mathrm{CNV}$ calls contributing to the significant $\mathrm{CNV}$ region (PenMaxP) $>0.5$ and high frequency (Freq $>0.5$ ), nearly identical segmental duplications (SegDups) > 10 , any locus frequently found in multiple studies such as T-cell receptor gene, human major histocompatibility complex gene etc. (Recurrent), the same inflated sample driving multiple CNV association signals (FreqInflated), the HMM confidence score in PennCNV calling (AvgConf) $<10$, and allele A or B banding (ABFreq) in BAF low for duplications. Additionally, if more than three of below criteria were met, the CNV also was not considered for further analysis: $\mathrm{CNV}$ residing at centromere or telomere regions (TeloCentro), high or low GC content regions (AvgGC $<30$ or $>60$ ), CNV regions with high population frequency (PopFreq) $>0.01$, a large gap in probe coverage exists within $\mathrm{CNV}$ association signals (Sparse) $>50 \mathrm{kbp}$, and average length of $\mathrm{CNV}<10$ $\mathrm{kbp}[27]$.

\section{CNV visualization, examination and laboratory validation} CNVs passing QC in both Groups 1 and 2 were examined by the plots of signal intensity (LRR/BAF) generated using the $\mathrm{CNV}$ visualization function implemented in the PennCNV package. Three CNVs passed $\mathrm{CNV}$ quality control in both groups and signal intensity visualization examination, and were chosen for further validation using TaqMan CNV probes located in the central region of each CNV (Hs04383175_cn, Hs06286795_cn and Hs06269635_cn), TaqMan CNV reference assay (human RNase P: 4403326) and QuantStudio 3D Digital PCR System (Thermo Fisher Scientific, Waltham, MA USA) by the Nemours Biomolecular Core Laboratory, following the manufacturer's standard protocol.

\section{Results}

Based on sample quality control criteria, 66 cases and 186 controls were removed, leaving 493 cases and 1586 controls in Group 1. In Group 2, 46 cases and 47 controls were removed, leaving 307 cases and 1102 controls.
In Group 1, 7,376 deletions and 4,313 duplications were detected and 6,689 deletions and 6,635 duplications were detected in Group 2.

In gene-based association tests, 25 and 106 genes/loci with deletion, and 371 and 177 genes/loci with duplication reached genome-wide significance $\left(p<5 \times 10^{-4}\right)$ in Group 1 and Group 2 (Additional file 1). After metaanalysis, 49 genes/loci with deletion and 331 genes/loci with duplication reached genome-wide significance (Additional file 2). For 49 genes with deletion, the direction of effect was consistent in the two groups. The direction of effect was inconsistent for 6 duplications and no direction was given in one duplication which was due to $p=1$ for that gene in gene-based association test of Group 2, and they were removed from further consideration, leaving 324 genes/loci with duplication. Among these 373 significant genes/loci, 17 with deletion and 1 with duplication were identified in CNV-based association analysis in both Group 1 and Group 2 (Table 1). Five genes/loci (TCR gamma alternate reading frame protein $(T A R P)$, tonsoku-like DNA repair protein (TONSL), TONSL antisense RNA 1 (TONSL-AS1), nucleobindin 2 (NUCB2), and UPF2 regulator of nonsense transcripts homolog (yeast) (UPF2)) with deletion passed CNV quality control in both groups (Table 1). Signal intensity plots of CNVs in NUCB2 and UPF2 (Fig. 1: Array plot of $\log \mathrm{R}$ ratio and $\mathrm{B}$ allele frequency for NUCB2 and UPF2) suggested heterozygous deletions: the LRR decrease below 0 and the BAF cluster around either 0 or 1 , but not near 0.5. Signal intensity plots of CNVs in TARP and TONSL/ TONSL-AS1 did not pass visualization examination (Additional file 3: Array plot of Log $\mathrm{R}$ ratio and $\mathrm{B}$ allele frequency for TARP and TONSL/TONSL-AS1) due to LRR close to 0 , BAF cluster near 0.5 , or both. Thus only CNVs in NUCB2 and UPF2 were further considered in our study.

The CNVs detected in NUCB2 are around $20 \mathrm{kbp}$ and $6.7 \mathrm{kbp}$ in Group 1 and Group 2, and they do not overlap. The Database of Genomic Variants (DGV) in The Hospital for Sick Children, a teaching hospital affiliated with the University of Toronto [30,31] reported a $15 \mathrm{kbp}$ deletion in 1 of 2026 individuals and a $719 \mathrm{bp}$ deletion in 2 of 2504 individuals at the CNV region of Group 1 (chr11:17300844-17320797), and a $5 \mathrm{kbp}$ deletion in 1 of 17421 individuals at the CNV region of Group 2 (chr11:17332461-17339127). Seven cases in Group 1 and 3 cases in Group 2 contained NUCB2 deletions based on this analysis. The CNVs detected in UPF2 were approximately $47.8 \mathrm{kbp}$ and $13 \mathrm{kbp}$ in Groups 1 and 2, respectively, and the $13 \mathrm{kbp}$ segment is inside the $47.8 \mathrm{kbp}$ segment. DGV reported a $47.8 \mathrm{kbp}$ deletion in 2 of 17421 individuals in this CNV region detected in Group 1. Four cases in Group 1 and 9 cases in Group 2 contained UPF2 deletions. The CNV confidence scores of NUCB2 and UPF2 for each case generated 


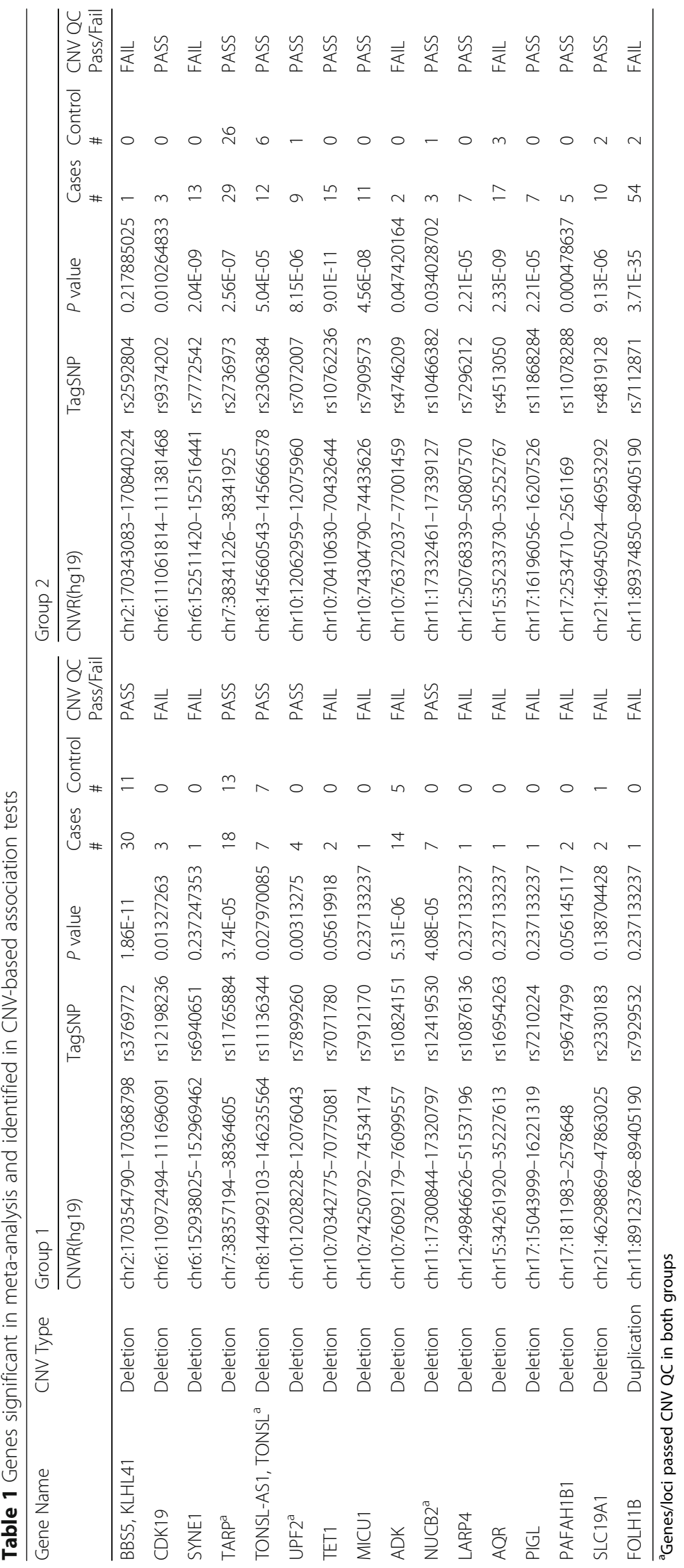




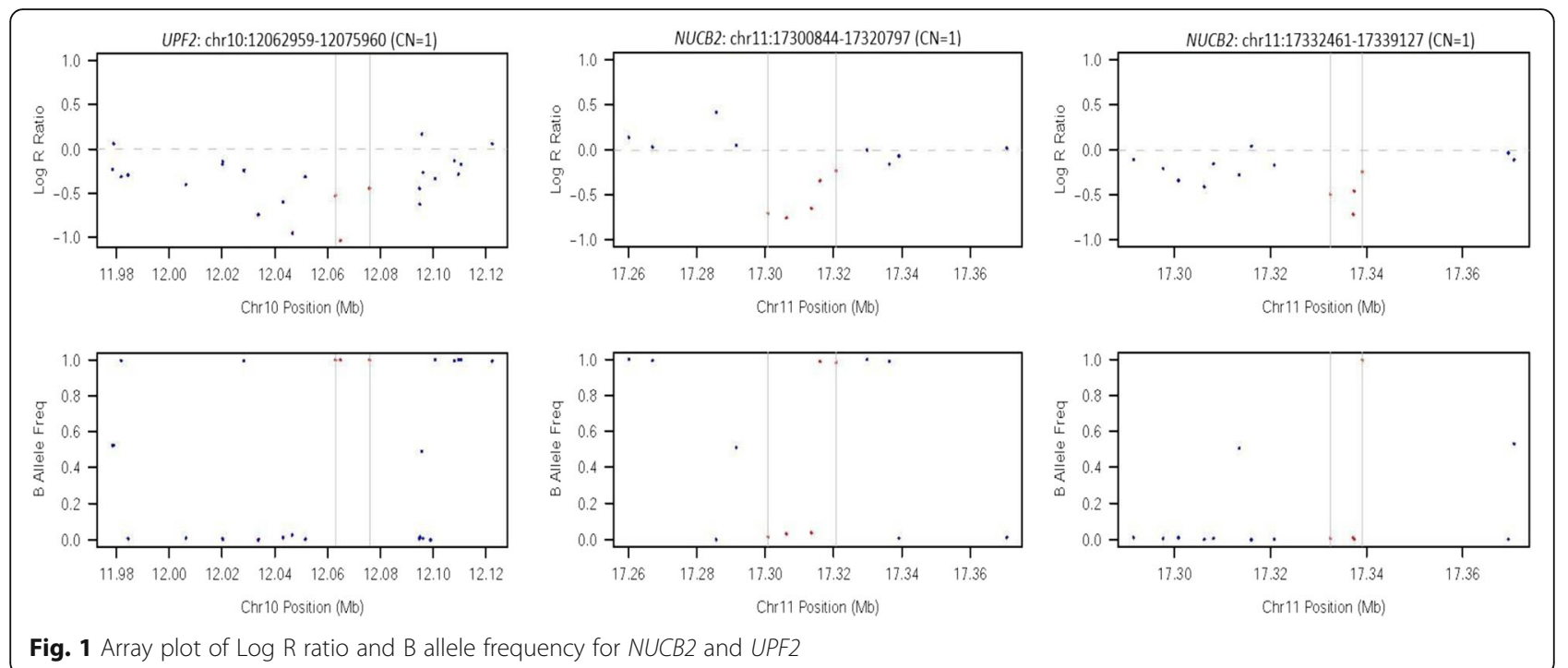

during CNV calling by PennCNV are shown in Table 2 . The score range was 12 to 55 , which is considered borderline reliable for $\mathrm{CNV}$ detection.

We attempted to validate CNVs in NUCB2 and UPF2 in affected cases using TaqMan CNV assays and QuantStudio $^{\circ}$ 3D Digital PCR System (Table 2). After validating the TaqMan CNV assays using 2 control DNAs without called CNVs in these regions, we tested 12 samples from Group 1 or 2 with called CNVs within these genes of interest (Table 2, noted in case IDs with underline). All 12 tested samples were diploid (Additional file 4), indicating that bioinformatically-called deletions were not validated by TaqMan CNV assays.

\section{Discussion}

Cryptorchidism is the most common male congenital genitourinary defect. While it is a manifestation of many congenital defect syndromes [32-34], the majority of cases are nonsyndromic and of unclear etiology. Our previous genome-wide association analyses of SNP data suggest that cryptorchidism is associated with significant genetic heterogeneity $[11,12]$. In the present study, we performed genome-wide $\mathrm{CNV}$ association analysis to identify the potential association of structural variation with the occurrence of nonsyndromic cryptorchidism, and our results suggest that CNVs do not contribute to the genetic basis of the nonsyndromic form of the disease.

In a previous report, Jorgez and colleagues identified a 2p15 deletion encompassing OTX1 in 6 subjects with genitourinary defects [15]. Three of these individuals had cryptorchidism and their genomic deletions also included EHBP1 and WDPC. Other genitourinary anomalies of the three patients with cryptorchidism were variable including absent prepuce, micropenis, discontinuous raphe, penile cyst, hypoplastic scrotum, kidney stones or small testes. The three patients also had other defects including developmental delay, vision problems and dysmorphic facial features. Structural variations were also identified in studies of subjects with nonobstructive azoospermia or congenital genitourinary tract masculinization disorders from the same research group $[14,35]$. In the study of nonobstructive azoospermia, 4

Table 2 Relevant validation information for genes (UPF2 and NUCB2) passed CNV QC and signal intensity examination

\begin{tabular}{|c|c|c|c|c|c|c|}
\hline Group & Gene Name & CNV (hg19) & $\begin{array}{l}\text { Probe \# in overlapping } \\
\text { CNV region }\end{array}$ & CNV size $(b p)$ & $\begin{array}{l}\text { TaqMan CNV } \\
\text { assays and location }\end{array}$ & $\begin{array}{l}\text { Cases with deletion } \\
\text { (CNV confidence score) }\end{array}$ \\
\hline \multirow[t]{2}{*}{ Group 1} & UPF2 & $\begin{array}{l}\text { chr10:12028228- } \\
12076043\end{array}$ & 10 & 47815 & $\begin{array}{l}\text { Hs04383175_cn; } \\
\text { Chr10:12063665 }\end{array}$ & $\underline{\mathrm{D} 10}(26)^{\mathrm{a}}, \underline{\mathrm{D} 29}(55)^{\mathrm{a}}, \mathrm{D} 34(46), 1495$ (27) \\
\hline & NUCB2 & $\begin{array}{l}\text { chr11:17300844- } \\
17320797\end{array}$ & 5 & 19953 & $\begin{array}{l}\text { Hs06286795_cn; } \\
\text { Chr11:17306162 }\end{array}$ & $\begin{array}{l}\mathrm{D} 8 \text { (24), } \mathrm{D} 10 \\
\mathrm{D} 33 \\
\text { (26), }\end{array}$ \\
\hline \multirow[t]{2}{*}{ Group 2} & UPF2 & $\begin{array}{l}\text { chr10:12062959- } \\
12075960\end{array}$ & 3 & 13001 & $\begin{array}{l}\text { Same as UPF2 } \\
\text { assay in group } 1\end{array}$ & $\begin{array}{l}7279(15), 7334(12)^{a}, 7338(23), 7339(16), \\
7341 \text { (14), } 7370(26)^{a}, \text {, } 7453(16), 7475 \text { (24), } \\
\underline{7479} \text { (17) }\end{array}$ \\
\hline & NUCB2 & $\begin{array}{l}\text { chr11:17332461- } \\
17339127\end{array}$ & 4 & 6666 & $\begin{array}{l}\text { Hs06269635_cn; } \\
\text { Chr11:17336218 }\end{array}$ & $\underline{7334}(17)^{\mathrm{a}}, \underline{7361}(14), \underline{7370}(12)^{\mathrm{a}}$ \\
\hline
\end{tabular}


patients with microduplications and 4 with microdeletions of E2F1 were identified among 110 affected individuals, but not among 78 fertile controls [35]. Two of the 8 patients with CNVs had cryptorchidism. Two nonsynonymous mutations of E2F1 (Ala102Thr and Gly393Ser) were also identified in three other patients, and one synonymous mutation (Leu415Leu) was identified in a patient with microduplication of E2F1. The patient with the Ala102Thr variant also had cryptorchidism. In the congenital genitourinary tract masculinization disorders study [14], copy number gains on Xq28 encompassing VAMP7 were found in 4 of 296 patients. Two of them had idiopathic cryptorchidism, and the other two had hypospadias. They also found 1 case of hypospadias with VAMP7 copy number gain in 28 distinct primary cultures of genital skin fibroblasts. All of the above three studies used array comparative genomic hybridization, a technology that enables efficient screening for CNVs, to discover the genomic variants. Other studies from Europe have also reported the microdeletions (2p14-p15, 2p15-16.1) in boys with cryptorchidism [36, 37]. However, all of these patients presented with other features besides cryptorchidism, including intellectual disability, developmental delay and/or dysmorphic features. In our study, subjects were excluded if there was evidence for other genital anomalies and/or other clinical features in addition to undescended testes.

Only autosomal CNVs were called and analyzed in our study, which may have led us to miss associated CNVs on the $\mathrm{X}$ or $\mathrm{Y}$ chromosome. The significant genes in our meta-analysis with CNVs that also passed QC in both groups and signal intensity visualization examination are NUCB2 and UPF2, located at chromosome 11 and chromosome 10, respectively. However, these deletions were not validated by QuantStudio ${ }^{\circ}$ 3D Digital PCR System with TaqMan CNV assays in our study samples, despite the signal intensity plots suggesting the presence of heterozygous deletions. The confidence score range of detected cases for these deletions is 12 to 55 (Table 2). The score numbers are lower in Group 2 cases with most of them less than 20. A confidence score of 10 has been suggested as a threshold to classify reliable CNV calls while the higher scores are more reliable and more likely to be replicated [38]. Most of our scores were less than the median score of 27.7 that was reported for deletions that could be replicated in the study of $\mathrm{Ku}$ et al. [38]. Due to different platforms with low overlapping SNP coverage that were used in genotyping Group 1 and Group 2 samples, we performed association tests separately in the two groups. Consequently, the whole study power was reduced compared to what it would have been if all samples had been genotyped on the same platform and some CNVs associated with disease may have been missed, even though we used meta-analysis to combine the two data sets. The use of SNP genotyping array data for CNV analysis is a common and acceptable approach [39-42], but the global CNV coverage of our SNP arrays varies. Cooper GM et al. [43] reported approximately $40 \%$ and $80 \% \mathrm{CNV}$ coverage for Illumina chips of HumanHap550 and Human 1 M. Besides HumanHap550, the other chips we used, Human610Quad and Human OminiExpress, have fewer SNPs compared to the Human $1 \mathrm{M}$, and therefore likely have less than $80 \%$ global CNV coverage. Cooper GM et al. also reported that only two-thirds of detected CNVs by SNP data from Human $1 \mathrm{M}$ could be validated in independent experiments [43], indicating that using SNP array data for $\mathrm{CNV}$ analysis may result in false positives, as may be the case in the present analysis.

\section{Conclusions}

A sample size (800 cases and 2688 controls) greater than that of any other $\mathrm{CNV}$ analysis of nonsyndromic cryptorchidism failed to identify any associated variants, but weak effects at multiple genomic loci may still contribute to the etiology of this disease. It is also possible that CNVs are present but were not detected due to insufficient coverage by the SNP arrays we used and/or, the present analysis was underpowered to identify rare, strong effect CNVs that contribute to disease risk. Whole genome or exome sequencing, and comparative genomic hybridization are alternative approaches for discovery of disease-associated SNPs and CNVs, but beyond the scope of the present studies. It is possible that structural variation is more commonly associated with syndromic cryptorchidism, but our inability to validate the candidate CNVs in this analysis suggests that these variants are not a major cause of nonsyndromic cryptorchidism.

\section{Additional files}

Additional file 1: genome-wide significant genes/loci in gene-based association tests in each group. Listed all the genes/loci with genomewide significance $\left(p<5 \times 10^{-4}\right)$ in gene-based association tests in each group and their $p$-values. (DOCX $55 \mathrm{~kb}$ )

Additional file 2: genome-wide signficant genes/loci in meta-analysis. Listed all the genes/loci with genome-wide significant $p$-values $\left(p<5 \times 10^{-4}\right)$ in meta-analyses of gene-based association results, and their z-scores, $p$-values and the direction of effect in the two groups. (DOCX $57 \mathrm{~kb}$ )

Additional file 3: Array plot of $L o g \mathrm{R}$ ration and $\mathrm{B}$ allele frequency for TARP and TONSL/TONSL-AS1. Showed the signal intensity plots of 2 CNVs in TARP and 1 CNV in TONSL/TONSL-AS1. The array plots did not pass visualization examination due to LRR close to 0, BAF cluster near 0.5, or both. (DOCX $141 \mathrm{~kb}$ )

Additional file 4: CNVs validation using TagMan CNV assays and QuantStudio ${ }^{\otimes}$ 3D Digital PCR System. Listed the samples tested for CNV validating and the results. (XLSX $10 \mathrm{~kb}$ )

Additional file 5: CNV calls for Group 1 cases passed sample QC. Each column in Additional file 5 represents CNV location, SNPs numbers contained within the CNV, the length of the CNV, copy number ( $\mathrm{cn}$ ) of the CNV call, sample id, the starting marker identifier and the ending marker identifier in the CNV, and confidence score in PennCNV calling. (XLSX 653 kb) 
Additional file 6: CNV calls for Group 1 controls passed sample OC. Each column in Additional file 6 represents CNV location, SNPs numbers contained within the CNV, the length of the CNV, copy number (cn) of the CNV call, sample id, the starting marker identifier and the ending marker identifier in the CNV, and confidence score in PennCNV calling. (XLSX $1544 \mathrm{~kb}$ )

Additional file 7: CNV calls for Group 2 cases passed sample QC. Each column in Additional file 7 represents CNV location, SNPs numbers contained within the CNV, the length of the CNV, copy number (cn) of the CNV call, sample id, the starting marker identifier and the ending marker identifier in the CNV, and confidence score in PennCNV calling. (XLSX $534 \mathrm{~kb}$ )

Additional file 8: CNV calls for Group 2 controls passed sample QC. Each column in Additional file 8 represents CNV location, SNPs numbers contained within the CNV, the length of the CNV, copy number (cn) of the CNV call, sample id, the starting marker identifier and the ending marker identifier in the CNV, and confidence score in PennCNV calling. (XLSX 1952 kb)

\section{Abbreviations}

BAF: B allele frequency; CHOP: The Children's Hospital of Philadelphia; CNV: Copy number variation; DGV: Database of genomic variants; GCWF: Guanine-cytosine base pair wave factor; GWAS: Genome-wide association study; HGDP: Human genome diversity project; LRR: Log R ratio; MDS: Multidimensional scaling; NUCB2: Nucleobindin 2; QC: Quality control; SD: Standard deviation; SDLRR: Standard deviation of log R ratio; SNP: Single Nucleotide polymorphisms; UPF2: UPF2 regulator of nonsense transcripts homolog (yeast)

\section{Acknowledgements}

The authors would like to thank all our participants and their families for their gracious participation in this study.

\section{Funding}

Study design, data collection, data analysis and interpretation, and writing the manuscript were supported by R01HD060769 from the Eunice Kennedy Shriver National Institute for Child Health and Human Development (NICHD), and Nemours Biomedical Research Fund. Part of data analysis and interpretation, and manuscript publication were supported by an Institutional Development Award (IDeA) from the National Institute of General Medical Sciences (NIGMS) of the National Institutes of Health under grant numbers P20GM103464 and P30GM114736 Center for Pediatric Research (COBRE) and grant P20GM103446 (DE-INBRE). Part of data collection and analysis was supported by an Institute Development Fund to the Center for Applied Genomics at The Children's Hospital of Philadelphia.

\section{Availability of data and materials}

The datasets supporting the conclusions of this article are included in Additional files 5, 6, 7 and 8 .

\section{Authors' contributions}

YW: sample preparation, data analysis and draft of manuscript; JL: data analysis; TFK, AOF, TEF, AHB, JAH, RG, PHN, RMC, KRH, DJA, JSB: recruitment, sample collection and phenotyping; DS: CNV laboratory validation; CEK: genotyping; KSC, HH, MD, JSB: study design. All authors read and approved the final manuscript.

\section{Competing interests}

The authors declare that they have no competing interests.

\section{Consent for publication}

Not applicable.

\section{Ethics approval and consent to participate}

Informed consent was obtained for all participants and studies were approved by the Institutional Review Board at Nemours/Alfred I. DuPont Hospital for Children (Nemours) or The Children's Hospital of Philadelphia (CHOP)

\section{Author details}

${ }^{1}$ Nemours Biomedical Research, Nemours /Alfred I. duPont Hospital for Children, Wilmington, DE 19803, USA. '2Division of Urology, Nemours/Alfred I. duPont Hospital for Children, Wilmington, DE 19803, USA. ${ }^{3}$ Center for Applied Genomics, The Children's Hospital of Philadelphia, Philadelphia, PA
19104, USA. Division of Urology, The Children's Hospital of Philadelphia, Philadelphia, PA 19104, USA. ${ }^{5}$ Division of Genetics, The Children's Hospital of Philadelphia, Philadelphia, PA 19104, USA. 'Department of Pediatrics, Perelman School of Medicine, University of Pennsylvania, Philadelphia, PA 19104, USA. ${ }^{7}$ Department of Biostatistics and Epidemiology, Perelman School of Medicine, University of Pennsylvania, Philadelphia, PA 19104, USA. ${ }^{8}$ Department of Molecular Medicine, Sapienza University, Rome, Italy. ${ }^{9}$ Present address: Auf der Bult Kinder- und Jugendkrankenhaus, Hannover, Germany. ${ }^{10}$ Present address: Division of Pediatric Urology, Cincinnati Children's Hospital Medical Center, Cincinnati, OH, USA.

Received: 22 October 2015 Accepted: 14 October 2016 Published online: 21 October 2016

\section{References}

1. Ashley RA, Barthold JS, Kolon TF. Cryptorchidism: pathogenesis, diagnosis, treatment and prognosis. Urol Clin North Am. 2010;37:183-93.

2. Schnack TH, Zdravkovic S, Myrup C, Westergaard T, Wohlfahrt J, Melbye M. Familial aggregation of cryptorchidism-a nationwide cohort study. Am J Epidemiol. 2008;167:1453-7.

3. Ferlin A, Zuccarello D, Zuccarello B, Chirico MR, Zanon GF, Foresta C. Genetic alterations associated with cryptorchidism. JAMA. 2008;300:2271-6.

4. Foresta C, Zuccarello D, Garolla A, Ferlin A. Role of hormones, genes, and environment in human cryptorchidism. Endocr Rev. 2008:29:560-80.

5. Bertini V, Bertelloni S, Valetto A, Lala R, Foresta C, Simi P. Homeobox HOXA10 gene analysis in cryptorchidism. J Pediatr Endocrinol Metab. 2004;17:41-5.

6. Galan JJ, Guarducci E, Nuti F, Gonzalez A, Ruiz M, Ruiz A, et al. Molecular analysis of estrogen receptor alpha gene AGATA haplotype and SNP12 in European populations: potential protective effect for cryptorchidism and lack of association with male infertility. Hum Reprod. 2007;22:444-9.

7. Kolon TF, Wiener JS, Lewitton M, Roth DR, Gonzales Jr ET, Lamb DJ. Analysis of homeobox gene HOXA10 mutations in cryptorchidism. J Urol. 1999;161:275-80

8. Wang Y, Barthold J, Kanetsky PA, Casalunovo T, Pearson E, Manson J. Allelic variants in HOX genes in cryptorchidism. Birth Defects Res A Clin Mol Teratol. 2007:79:269-75.

9. Wang Y, Barthold J, Figueroa E, Gonzalez R, Noh PH, Wang M, et al. Analysis of five single nucleotide polymorphisms in the ESR1 gene in cryptorchidism. Birth Defects Res A Clin Mol Teratol. 2008;82:482-5.

10. Yoshida R, Fukami M, Sasagawa I, Hasegawa T, Kamatani N, Ogata T. Association of cryptorchidism with a specific haplotype of the estrogen receptor alpha gene: implication for the susceptibility to estrogenic environmental endocrine disruptors. J Clin Endocrinol Metab. 2005:90:4716-21.

11. Barthold JS, Wang Y, Kolon TF, Kollin C, Nordenskjöld A, Olivant Fisher A, et al. Phenotype specific association of the TGFBR3 locus with nonsyndromic cryptorchidism. J Urol. 2015;193:1637-45

12. Barthold JS, Wang Y, Kolon TF, Kollin C, Nordenskjöld A, Olivant Fisher A, et al. Pathway analysis supports association of nonsyndromic cryptorchidism with genetic loci linked to cytoskeleton-dependent functions. Hum Reprod. 2015:30:2439-51.

13. Mikhail FM. Copy number variations and human genetic disease. Curr Opin Pediatr. 2014;26:646-52.

14. Tannour-Louet M, Han S, Louet JF, Zhang B, Romero K, Addai J, et al. Increased gene copy number of VAMP7 disrupts human male urogenital development through altered estrogen action. Nat Med. 2014;20:715-24.

15. Jorgez CJ, Rosenfeld JA, Wilken NR, Vangapandu HV, Sahin A, Pham D, et al. Genitourinary defects associated with genomic deletions in 2p15 encompassing OTX1. PLoS One. 2014; doi:10.1371/journal.pone.0107028

16. Li M, Li C, Guan W. Evaluation of coverage variation of SNP chips for genome-wide association studies. Eur J Hum Genet. 2008;16:635-43.

17. Wang K, Li M, Hadley D, Liu R, Glessner J, Grant SF, et al. PennCNV: an integrated hidden Markov model designed for high-resolution copy number variation detection in whole-genome SNP genotyping data. Genome Res. 2007;17:1665-74

18. Wang K, Chen Z, Tadesse MG, Glessner J, Grant SF, Hakonarson H, et al. Modeling genetic inheritance of copy number variations. Nucleic Acids Res. 2008; doi:10.1093/nar/gkn641

19. Diskin SJ, Li M, Hou C, Yang S, Glessner J, Hakonarson H, et al. Adjustment of genomic waves in signal intensities from whole-genome SNP genotyping platforms. Nucleic Acids Res. 2008; doi:10.1093/nar/gkn556 20. PennCNV. http://penncnv.openbioinformatics.org/en/latest/. Accessed 9 Oct 2013. 
21. Purcell S, Neale B, Todd-Brown K, Thomas L, Ferreira MA, Bender D, et al. PLINK: a tool set for whole-genome association and population-based linkage analyses. Am J Hum Genet. 2007;81:559-75.

22. PLINK v1.07. http://pngu.mgh.harvard.edu/purcell/plink/. Accessed 7 May 2011 .

23. Anderson CA, Pettersson FH, Clarke GM, Cardon LR, Morris AP, Zondervan KT. Data quality control in genetic case-control association studies. Nat Protoc. 2010;5:1564-73.

24. Rosenberg NA. Standardized subsets of the HGDP-CEPH Human Genome Diversity Cell Line Panel, accounting for atypical and duplicated samples and pairs of close relatives. Ann Hum Genet. 2006;70:841-7.

25. Human Genome Diversity Project. http://www.hagsc.org/hgdp/files.html. Accessed 19 July 2013.

26. Glessner JT, Li J, Hakonarson H. ParseCNV integrative copy number variation association software with quality tracking. Nucleic Acids Res. 2013; doi:10.1093/nar/gks1346

27. ParseCNV. http://parsecnv.sourceforge.net/. Accessed 10 Nov 2014

28. Willer CJ, Li Y, Abecasis GR. METAL: fast and efficient meta-analysis of genomewide association scans. Bioinformatics. 2010;26:2190-1.

29. METAL. http://csg.sph.umich.edu/abecasis/metal/. Accessed 2 Apr 2015.

30. MacDonald JR, Ziman R, Yuen RK, Feuk L, Scherer SW. The database of genomic variants: a curated collection of structural variation in the human genome. Nucleic Acids Res. 2014:42:D986-92. doi:10.1093/nar/gkt958.

31. Database of Genomic Variants. http://dgv.tcag.ca/dgv/app/home. Accessed 28 June 2015

32. Neuhann TM, Müller D, Hackmann K, Holzinger S, Schrock E, Di Donato N. A further patient with van Maldergem syndrome. Eur J Med Genet. 2012:55:423-8.

33. Lipska BS, Brzeskwiniewicz M, Wierzba J, Morzuchi L, Piotrowski A, Limon J. $8.6 \mathrm{Mb}$ interstitial deletion of chromosome $4 q 13.3 q 21.23$ in a boy with cognitive impairment, short stature, hearing loss, skeletal abnormalities and facial dysmorphism. Genet Couns. 2011;22:353-63.

34. Fei X, Qi M, Zhao Y, Li-Ling J. Identification and characterization of a complex pure mosaic of small supernumerary marker chromosomes involving $11 \mathrm{p} 11.12 \rightarrow \mathrm{q} 12.1$ and $19 \mathrm{p} 12 \rightarrow \mathrm{q} 12$ regions in a child featuring multiple congenital anomalies. Am J Med Genet A. 2011;155A:3116-21.

35. Jorgez CJ, Wilken N, Addai JB, Newberg J, Vangapandu HV, Pastuszak AW, et al. Genomic and genetic variation in E2F transcription factor-1 in men with nonobstructive azoospermia. Fertil Steril. 2015:103:44-52.

36. Hancarova M, Vejvalkova S, Trkova M, Drabova J, Dleskova A, Vlckova M, et al. Identification of a patient with intellectual disability and de novo $3.7 \mathrm{Mb}$ deletion supports the existence of a novel microdeletion syndrome in 2p14-p15. Gene. 2013;516:158-61.

37. Piccione M, Piro E, Serraino F, Cavani S, Ciccone R, Malacarne M, et al. Interstitial deletion of chromosome 2p15-16.1: report of two patients and critical review of current genotype-phenotype correlation. Eur J Med Genet. 2012:55:238-44.

38. Ku CS, Pawitan Y, Sim X, Ong RT, Seielstad M, Lee EJ et al. Genomic copy number variations in three Southeast Asian populations. Hum Mutat. 2010;31:851-7.

39. Dajani R, Li J, Wei Z, Glessner JT, Chang X, Cardinale CJ, et al. CNV Analysis Associates AKNAD1 with Type-2 Diabetes in Jordan Subpopulations. Sci Rep. 2015;5:13391.

40. Li J, Fung I, Glessner JT, Pandey R, Wei Z, Bakay M, et al. Copy Number Variations in CTNNA3 and RBFOX1 Associate with Pediatric Food Allergy. J Immunol. 2015;195:1599-607.

41. Peiffer DA, Le JM, Steemers FJ, Chang W, Jenniges T, Garcia F, et al. Highresolution genomic profiling of chromosomal aberrations using Infinium whole-genome genotyping. Genome Res. 2006;16:1136-48,

42. Komura D, Shen F, Ishikawa S, Fitch KR, Chen W, Zhang J, et al. Genomewide detection of human copy number variations using high-density DNA oligonucleotide arrays. Genome Res. 2006:16:1575-84.

43. Cooper GM, Zerr T, Kidd JM, Eichler EE, Nickerson DA. Systematic assessment of copy number variant detection via genome-wide SNP genotyping. Nat Genet. 2008:40:1199-203.

\section{Submit your next manuscript to BioMed Central and we will help you at every step:}

- We accept pre-submission inquiries

- Our selector tool helps you to find the most relevant journal

- We provide round the clock customer support

- Convenient online submission

- Thorough peer review

- Inclusion in PubMed and all major indexing services

- Maximum visibility for your research

Submit your manuscript at www.biomedcentral.com/submit
Biomed Central 